2018-08

\title{
Assessing children's interpersonal emotion regulation with virtual agents: The serious game Emodiscovery
}

\section{Pacella, D}

http://hdl.handle.net/10026.1/12290

\subsection{6/j.compedu.2018.04.005}

Computers \& Education

Elsevier

All content in PEARL is protected by copyright law. Author manuscripts are made available in accordance with publisher policies. Please cite only the published version using the details provided on the item record or document. In the absence of an open licence (e.g. Creative Commons), permissions for further reuse of content should be sought from the publisher or author. 
\& Education

Elsevier Editorial System(tm) for Computers

Manuscript Draft

Manuscript Number: CAE-D-17-00895R1

Title: Assessing Children's Interpersonal Emotion Regulation with Virtual Agents: The Serious Game Emodiscovery

Article Type: Research Paper

Keywords: intelligent tutoring systems; interactive learning environments; simulations; applications in subject areas; architectures for educational technology system

Corresponding Author: Miss Daniela Pacella,

Corresponding Author's Institution: Plymouth University

First Author: Daniela Pacella

Order of Authors: Daniela Pacella; Belén López-Pérez

Abstract: Emotion regulation (ER) is key for children's development and it has recently been considered in many serious games and e-learning technologies. However, these tools have focused on children's efforts to change their own emotions (intrapersonal ER), overlooking how children may engage in modifying the emotions of others (interpersonal ER). To address this gap, we have developed a multiplatform online serious game, Emodiscovery, which evaluates whether children (8-11 year-olds) tend to use different adaptive and maladaptive regulation strategies to change others' negative emotions with the use of 3D animated characters. The game is organized into levels, each representing a different scenario, where the child is asked to interact with the character three times choosing an appropriate regulation strategy to cheer him or her up from four possible options, being two adaptive and two maladaptive strategies. To test the effectiveness of the game, a sample of English children played three scenarios depicting a character feeling sadness, anger, and fear, respectively. Results showed that most children chose adaptive strategies to improve the character's emotion across the different emotion scenarios. Furthermore, emotion recognition was not positively related to children's choices of regulation strategies. The implications and applications of the games are discussed. 
Editorial Board

Computers and Education

Plymouth, 22 $2^{\text {nd }}$ June, 2017

Dear Drs Tsai, Heller, and Nussbaum,

We have the pleasure of sending you our manuscript entitled, "Assessing children's interpersonal emotion regulation with virtual agents: the serious game Emodiscovery" (7323 words, whole document). This research presents a newly developed serious game to investigate - and with the potential to train - children's efforts to modify others' emotions (i.e., interpersonal emotion regulation).

Emotion regulation (ER) consists of different processes aimed at initiating, changing, or inhibiting one's own (intrapersonal) or others' (interpersonal) emotional responses (Gross, 2007). ER constitutes a key variable in the study of children's development (Eisenberg, 2000), as the use of maladaptive regulation strategies is a strong predictor of psychological disorders (e.g., Southam-Gerow \& Kendall, 2002), low social adjustment (e.g., Eisenberg \& Fabes, 2006), poor academic performance (e.g., Blair, 2002), and ineffective interpersonal functioning across the lifespan (e.g., Richards \& Gross, 2000). Although most research has been conducted on intrapersonal ER (e.g., Eisenberg, 2000; Gross, 2007), more recently researchers have started looking at interpersonal ER (e.g., Zaki \& Williams, 2013) suggesting different classifications of regulation strategies (e.g., Niven, Totterdell, \& Holman, 2009; Williams, 2007).

This preference for intrapersonal ER has also been observed in the development and testing of serious games. For example, in Game Teen (Rodriguez et al., 2012) adolescents could learn through playing two different levels how to regulate their own joy and frustration. Other games have been developed to train people how to regulate their own levels of arousal (i.e., Auction game; Jercic, Astor, Adam, \& Hilborn, 2012; and NeuroIS; Astor, Adam, Jerčić, Schaaff, \& Weinhardt, 2014; The Island VG; Konstantas et al., 2013). Although these games have been a good first step in the development of serious games to evaluate and train people's ER, they have been focused exclusively in the intrapersonal domain leaving a gap in the interpersonal ER domain.

Emodiscovery has been developed to address this gap focusing on children between 8-11 year of age. In this paper we present the different pilots conducted for the development of the different scenarios, as well as the first test conducted in typically developing children (58 children; 37 boys and 21 girls; 8 to 11 year-olds, $M_{\text {age }}=8.81$ ). The results of the application showed that children may find more difficult to accurately identify the emotions of anger and fear, compared to sadness. This is in line with previous findings that showed how children (from 8 to 10 years) find it more difficult to recognize the emotions of anger and fear compared to sadness (Sauter, Panattoni, \& Happé, 2012). Regarding the use of regulation strategies, most children used an adaptive strategy to change the emotion of the character in the story. Unlike expected, there was not an interaction between accurately 
identifying the emotion felt by the character and the selection of an adaptive strategy. Finally, children showed consistency throughout the scenarios. Thus, children who tended to choose an adaptive strategy to improve the character's sadness did choose an adaptive strategy to improve the character's anger and fear. This is in line with previous research on the use of regulation strategies that showed that, overall, people tend to use similar strategies across contexts (Niven, Macdonald, \& Holman, 2012).

Although the present research constitutes only preliminary evidence of the game, we believe Emodiscovery can be extremely beneficial to advance our knowledge about children's interpersonal emotion regulation and to promote better and more tailored interventions.

The findings reported in our manuscript have not been previously published and the manuscript is not being simultaneously submitted elsewhere. Research was conducted in compliance with APA and BPS ethical standards in the treatment of participants.

We hope that this research will meet the requirements of your journal, and we remain at your full disposal for any further questions.

Sincerely,

Daniela Pacella

School of Computing, Electronics and Mathematics, Plymouth University, UK

pacelladaniela@gmail.com

PL4 8AA

Telephone: 00393398919356

Dr. Belén López-Pérez

Department of Psychology, Liverpool Hope University, UK

lopezpb@hope.ac.uk

Faculty of Science

L16 9JD

Telephone: 01512913832 
Editorial Board

Computers and Education

Dear Dr. Heller and reviewers,

We would like to thank you for the thoughtful and interesting comments provided during the revision of the manuscript with title “Assessing Children's Interpersonal Emotion Regulation with Virtual Agents: The Serious Game Emodiscovery”.

We carefully analysed the reviewers' comments and, in response, implemented the following modifications:

Reviewer \#2: There is also a flaw though which the authors really need to address: a substantial number of the in-text references is missing from the reference list, e.g. Richards \& Gross, 2000; Jercic et al. 2012; Astor et al. 2014; Konstantas et al. 2013; Polson, 1988; Batson, 2011; Wickens, 1989; Sauter et al. 2012; Grynszpan et al. 2008.

We agree with the reviewer that, in the submission process, a number of references in the section went missing. All of the missing citations have now been correctly included in the References section.

Reviewer \#3: I know I might be a bit old fashioned, but I still find the structural developmental levels of Piaget helpful in assessing cognitive interactions and abilities. Not a major issue, really, just a minor quibble of mine. By and large, nice work.

We thank the reviewer for their compliment about our work. We have included some discussion of the results in light with Piaget's theory (1983). We have now included discussion on the concepts of assimilation and accommodation. We have also discussed how learning may happen at the concrete operational stage (according to the age range of our sample). It reads as follows (pages 7 and 8):

"Given that Emodiscovery has the potential to provide feedback, one of its possible direct applications can be fostering children's interpersonal ER skills in educational contexts. Following Piaget's theory (1983), the feedback provided could help children to accommodate their learning on interpersonal ER, as it will help them to change their potential maladaptive responses to more adaptive ones. Furthermore, the existence of different levels with distinct emotions (i.e., sadness, anger, and fear) may help children to assimilate the different regulation strategies (i.e., fitting the new regulation strategies in their previous schemas of how to cheer up others) and use them across different levels. The game was designed for children between 8 to 11 years of age, which means they are capable of abstract problem solving (concrete operational stage; Piaget, 1983); hence, it is not surprising 
that children could represent the different emotional situations and provided in most cases a successful response by choosing an adaptive regulation strategy. Although in this first evaluation of the game we did not assess if children were capable of applying their learning (i.e., use of adaptive regulation strategies to other new contexts or levels); according to Piaget's theory (1983), learning is likely to happen as children showed active engagement. In fact, the design of the game with an online peer should be a major driving force for learning as well”.

We hope we have addressed the comments appropriately and that our paper is suitable for publication at Computers and Education.

Sincerely,

The authors 
Assessing Children's Interpersonal Emotion Regulation with Virtual Agents: The Serious Game Emodiscovery

\author{
Daniela Pacella ${ }^{1} \&$ Belén López-Pérez ${ }^{2}$ \\ ${ }^{1}$ School of Computing, Electronics and Mathematics, Plymouth University, UK \\ ${ }^{2}$ Department of Psychology, Liverpool Hope University, UK
}

*Correspondence should be addressed to Daniela Pacella (School of Computing, Electronics and Mathematics, Plymouth University, Drake Circus, PL4 8AA, Plymouth, UK)

Email address: pacelladaniela@gmail.com 


\begin{abstract}
Emotion regulation (ER) is key for children's development and it has recently been considered in many serious games and e-learning technologies. However, these tools have focused on children's efforts to change their own emotions (intrapersonal ER), overlooking how children may engage in modifying the emotions of others (interpersonal ER). To address this gap, we have developed a multiplatform online serious game, Emodiscovery, which evaluates whether children (8-11 year-olds) tend to use different adaptive and maladaptive regulation strategies to change others' negative emotions with the use of 3D animated characters. The game is organized into levels, each representing a different scenario, where the child is asked to interact with the character three times choosing an appropriate regulation strategy to cheer him or her up from four possible options, being two adaptive and two maladaptive strategies. To test the effectiveness of the game, a sample of English children played three scenarios depicting a character feeling sadness, anger, and fear, respectively. Results showed that most children chose adaptive strategies to improve the character's emotion across the different emotion scenarios. Furthermore, emotion recognition was not positively related to children's choices of regulation strategies. The implications and applications of the games are discussed.
\end{abstract}

Keywords: intelligent tutoring systems; interactive learning environments; simulations; applications in subject areas; architectures for educational technology system. 
1 Introduction

Emotion regulation (ER) consists of different processes aimed at initiating, changing, or inhibiting one's own (intrapersonal) or others' (interpersonal) emotional responses (Gross, 2007). ER constitutes a key variable in the study of children's development (Eisenberg, 2000), as the use of maladaptive regulation strategies is a strong predictor of psychological disorders (e.g., Southam-Gerow \& Kendall, 2002), low social adjustment (e.g., Eisenberg \& Fabes, 2006), poor academic performance (e.g., Blair, 2002), and ineffective interpersonal functioning across the lifespan (e.g., Richards \& Gross, 2000).

\subsection{Interpersonal Emotion Regulation}

Although most research has been conducted on intrapersonal ER (e.g., Eisenberg, 2000; Gross, 2007), more recently researchers have started looking at interpersonal ER (e.g., Zaki \& Williams, 2013) suggesting different classifications of regulation strategies (Niven, Totterdell, \& Holman, 2009; Williams, 2007). Adaptive interpersonal affect improvement strategies may comprise situation modification (i.e., removing or altering a problem to reduce its emotional impact) and attentional deployment (i.e., directing the target's attention to something more pleasant) (Little, Kluemper, Nelson, \& Gooty, 2012), as well as affective engagement (i.e., engaging directly with the target's feelings through listening, talking about the target's situation, reminding the target they did fine before, etc.), cognitive engagement (i.e., changing the way a target thinks about a situation through highlighting others' support, rationalizing, etc.), humour (i.e., improving the target's mood through acting silly, laughing, etc.) and attention (i.e., any action that implies giving consideration to the target) (Niven et al., 2009). Conversely, maladaptive interpersonal emotion regulation strategies may comprise co-rumination (i.e., extensively discussing others' problems with a focus on negative feelings which may increase worry rather than problem-solving; Parkinson \& Simons, 2012), avoidance (i.e., evading negative feelings and thoughts), or reinforcement of avoidance in others (i.e., suggesting others to evade negative feeling and thoughts; Mazefsky, Borue, Day, \& Minshew, 2014), and emotional suppression (i.e., inhibiting others' emotional response; Little et al., 2012).

\subsection{Development of Interpersonal Emotion Regulation}

Previous research has shown that interpersonal ER develops with age, as changing someone's mood requires not only the ability to represent another's emotional state but also to identify with them and discriminate between different emotional experiences (Dunfield, 2014). Although these skills are present since the age of three, children start generalizing them to other emotions (i.e., anger, fear, or surprise) at the age of five (Widen \& Russell, 2003). Furthermore, the use of cognitive strategies such as cognitive engagement is present mainly in 8 year-olds (López-Pérez, Wilson, Dellaria, \& Gummerum, 2016). Although previous research has identified age differences in interpersonal ER, prior studies have been conducted through observing children interacting with upset targets (e.g., Dunfield, O'Connell, Kuhlmeier, \& Kelley, 2011) or by asking children how they would react when facing an upset target (López-Pérez et al., 2016). Thus, it has not been evaluated (1) whether children's identifications of others' emotions may affect their strategies selection and (2) whether the regulation strategies selected may differ depending on the emotion displayed by the target. Consequently, the development of an online game for that purpose can be extremely useful.

\subsection{Serious Games on Emotion Regulation}

Concerning ER, different serious games have been developed with the aim of providing training to the users. Game Teen (Rodriguez et al., 2012) was one of the first games created for that purpose. In Game Teen, adolescents could play two levels (i.e., about joy and frustration) and were asked to navigate a character through several scenarios where they could learn the use of different regulation strategies dealing with positive or negative emotions.

Other games have been developed to investigate the individual's ability to control their own levels of arousal. Among these games, some have been developed focusing on the domain of economical decisionmaking (i.e., Auction game; Jercic, Astor, Adam, \& Hilborn, 2012; and NeuroIS; Astor, Adam, Jerčić, Schaaff, \& Weinhardt, 2013) whereas others have focused on clinical populations, such as patients suffering from Bulimia Nervosa (i.e., The Island VG; Fagundo et al., 2013).

Although these games represent a good first step in the development of serious games to evaluate and train people's ER, their focus lies exclusively in the intrapersonal domain, leaving a gap in the interpersonal ER domain.

\subsection{The Present Research}

The aim of the present research is to test Emodiscovery, a newly developed serious game focused on interpersonal ER. Emodiscovery is, to the best of our knowledge, the first 3D simulation game on 
interpersonal emotion regulation and specifically developed for children between the age of 8 and 11 . When designing the game, typically developing and high-functioning autistic children were chosen as the main target population. Concerning typically developing children, the game is thought to be used in educational contexts to promote socio-emotional skills within the classroom setting, as currently done with other games in the emotion education domain (Hromek \& Roffey, 2009). Regarding children with high-functioning autism, the game can be used in educational and therapeutic contexts to explore whether children experience any difficulty when interacting with others. Considering that the game includes a tutoring aspect and provides feedback on the user's response, it may be also used to promote socioemotional skills, as done with other games in this clinical population (Pioggia, Igliozzi, Sica, Ferro, Muratori, Ahluwalia, \& De Rossi, 2008).

\section{Method}

\subsection{Game Implementation Description}

\subsubsection{The Architecture of Emodiscovery}

Emodiscovery is a simulation-based game that allows the player to interact with peers represented by virtual characters that resemble children around the age of 10 and describes scenarios of real-life situations. Two versions of the game Emodiscovery were developed: a public version, freely accessible at the website http://emodiscovery.com, and an adapted version used exclusively by participants taking part in the study. The two versions differed only in few features, which will be described along with the structure of the software. The main components of the platform Emodiscovery are the user interface and the user model.

The user interface allows the interaction between the user and the software and it represents the graphics, the controls and the possible actions that the user can perform. It is thought intuitive, accessible and easy to understand for children, as it relies on the typical basic principles of usability of mobile and educational games (Amory, 2001; Shneiderman, 2010). The learner or user module represents the way the user is evaluated and the quantity and type of variables that are stored during the interaction with the interface. The user module is also in charge to interpret all the data obtained from the user in order to assess their performance. The evaluation provided is called learner's diagnosis (Polson, 2013).

\subsubsection{The User Interface}

Emodiscovery is a 3D simulation game and is accessible from different types of devices: PCs, using an executable program, or from mobile devices equipped with Android or iOS. A demo of the game is also available on the website in the HTML5 format. All released versions have the same interface and, except for the registration phase, require only mouse/touch input for the full interactive experience. For simple point and select tasks, in fact, research showed that mouse is the preferred input device and achieves the best performance when compared to other remote pointing devices (Natapov, Castelucci, \& MacKenzie 2009). For testing, therefore, the PC version was used, since only the use of a mouse is required and touchpads showed increased movement time and lower performance in comparison (Akamatsu \& MacKenzie, 2002). The user interface was created with the 3D game engine Unity and included 2D and 3D objects. Meshes, materials and animations for the 3D models were built with Blender.

Once the game is loaded, the home screen requests users to log in or register if they did not have a previous account. Users are welcomed by an owl, which is a neutral tutoring character inside the game who provides feedback. New users then reach a registration screen in which they are asked to enter a username, their age, gender and nationality, as shown in Figure 1. The game allows user identification and registration with the use of a cloud database. The BaaS service allows data stored to be safe and encrypted. The registration interface is available in English, Polish and Spanish and it is possible to instantly change the displayed language by clicking on the corresponding flag. Once the mandatory information has been inserted, the user is redirected to the level selection screen.

The level selection screen shows all the levels and the respective score achieved, represented by the amount of stars gained in each level. Levels that are available to play are colored while the others are greyed out. The availability of the levels depends on the progression of the player. In the public version, levels are progressively unlocked when the player has gained at least five stars in the previous level. In case of the version used for experimental purposes, each level is unlocked just by successfully completing the previous one, regardless of the number of stars obtained. At the current state of software development, there are six possible levels (two levels for each emotion displayed by the main character: sadness, anger, and fear). Each level contains a different scenario, describes a new situation, and introduces a new character. The logic about the flow of the scenarios is shown in Figure 2. 
The level interface shows the 3D character on the right, either a boy or a girl, a speech bubble near the head of the character, a text box on the left, containing the information delivered to the user and, on top, the "exit", "options" and "help" buttons. A screenshot of the interface inside the level is shown in Figure 3. There is no interactable object in the environment or on the background to reduce the possibility of distractions. At the beginning of level 1, the user is presented a brief instruction about the scope of the game and is explained how to interact with the character. This information is always available to view in the "help" section. At the beginning of all levels, there is a description about what happened to the main character. The character's gender is always matched to the gender of the user, as previous research has found that perspective taking and hence emotion recognition is facilitated (Batson, 2011). Depending on the level, the character's facial expression shows one out of the three negative emotions (i.e., sadness, fear, or anger). An example of the facial emotion expressions in the male characters can be found in Figure 4. After the brief introduction, the character also displays a brief sentence in its speech bubble to indirectly remark its emotional state. After that, the user is prompted with a question to indicate what emotion the character is feeling. The user is then provided with five possible answers, corresponding to five basic emotions (i.e., anger, fear, surprise, sadness and happiness). The sequence of the emotions that the character is displaying is fixed among levels, in particular scenarios 1 and 4 deal with the emotion of sadness, 2 and 5 with the emotion of anger and 3 and 6 with the emotion of fear. If the user selects the correct answer, a positive feedback is provided by the owl and displayed on top of the screen; otherwise, the user is invited to try again. If, on their second attempt, the user guesses correctly, a positive feedback is given, otherwise, the correct answer is provided with an explanation.

At the end of this phase, the user is asked to interact three times directly with the character to try to improve the character's emotions. Thus, the character displays a comment in regard to what happened to him/her and then the user is presented four possible sentences which represent two adaptive (e.g., reappraisal of the character's problem) and two maladaptive regulation strategies (e.g., co-ruminate with the character about his/her problem). Like in the initial question, the user has two chances to choose one of the adaptive strategies. To avoid users, that is children, to feel distressed by not being able to cheer up the character, at the end of the interactions the character always displays a happy facial expression and states that $\mathrm{s} / \mathrm{he}$ is feeling much better. Afterwards, the tutoring character appears and asks the player to take action. The user is asked to proceed to the next level - in case of the public version, to try the level again if the minimum score was not reached - or to go back to the level selection screen. If the user decides to terminate the scenario earlier using the "exit" button, a popup asks for confirmation and alerts the user that the progress will not be saved. An example of the full interaction is shown in Appendix A. The complete structure of the game is shown in Figure 5.

\subsubsection{The User Model}

The user variables recorded are different between the public and the experimental version and are summarized in Table 1. The difference lies in the data recorded at each level. In particular, response times were an important data to take into account while testing different populations but are not indicative for casual players, since interferences cannot be controlled. Also, while for clinical populations (e.g., children with high-functioning autism) it is important to take into account only the first time a scenario is played, for casual players the number of times a scenario is played helps evaluate the level of engagement and represents an important variable, therefore it is useful to collect data about consequent visits and use this information to personalize the user experience. The score gained in each level is shown in the level selection screen and measured as number of stars gained with a maximum of eight, if all the answers given at the first attempt are correct. Regarding the responses which are provided by the users inside each level there are three possible combination of answers for the initial question that explores the ability of the user to recognize another's emotion:

a) The user chooses the right emotion (2 stars)

b) The user chooses the wrong emotion and then the right emotion (1 star)

c) The user chooses two times a wrong emotion (0 stars)

For the consequent interactions that involve the direct dialogue (i.e., interpersonal emotion regulation) between the player and the character, there are four possible combinations:

a) The user chooses an adaptive regulation strategy ( 2 stars)

b) The user chooses a maladaptive and then an adaptive regulation strategy (1 star)

c) The user chooses a maladaptive and then a different maladaptive regulation strategy (0 stars)

d) The user chooses the same maladaptive strategy twice (0 stars)

If the answer provided the first time is not correct, the tutoring character will simply ask to provide another answer without adding more information. In case of possibility c), the tutoring character will provide more information about the emotion that the child is displaying, while in case of possibility d), more information is provided about why the chosen strategy is not efficient. 
2.2 Piloting of the Scenarios

The three scenarios used to test the game were previously piloted with a sample of 60 children (29 boys and 31 girls $)$ aged 8 to $11(\mathrm{M}=9.72, \mathrm{SD}=1.08)$ from the Southwest of England. Children's parents provided consent before children participated in the study. Children completed the study individually and they were read six stories (Appendix B) in a randomized order, matching the character to the child's gender. After listening to each story, each child was asked to identify how the character of the story was feeling and select one of the six possible options (happiness, sadness, anger, surprise, fear, and disgust). Only those stories in which the right emotion was identified by at least $75 \%$ of the sample were retained, as we wanted to ensure that children's regulation strategies choices may not be driven by not being able to accurately identify the emotion felt by the character in the story. Results showed that three out of the six stories were accurately identified by at least $75 \%$ of children (Table 2 ) and thus were retained.

\subsection{Possible Interaction Responses}

For each scenario, one author of the paper and a research assistant developed four possible interaction responses taking into account previous research on interpersonal emotion regulation, considering adaptive and maladaptive regulation strategies. Two experts on emotion regulation independent to the present research were asked to evaluate the different response interactions and indicate for each of them whether they portrayed an adaptive or a maladaptive regulation strategy and the subtype (see Appendix C).

For the scenario depicting sadness, the response interaction "I will tell you a joke" was not classified as expected by the experts. Although humor is an adaptive regulation strategy (see Appendix C), in this situation it was originally thought to possibly convey a maladaptive regulation strategy as it did not help to meet the target's emotion needs. Given that the two experts had different opinions on this, we decided to withdraw this possible interaction and substitute it by "Forget Whiskers, you can play with any other pet animal" which was correctly classified by the experts as maladaptive (general) and avoidance (subtype of regulation strategy).

For the scenario depicting anger, the response interaction "you're right" was not classified as maladaptive, counter to what it was expected. One of the experts interpreted this option as re-assurance. To make sure this could be interpreted as co-rumination it was rephrased to "you're right, you're not going to have time to make another card". After making this change, both experts classified it as maladaptive (general) and co-rumination (subtype of regulation strategy).

Finally, for the scenario depicting fear, the response interaction "you should study the lines more" was also initially classified as adaptive when it was intended to depict a maladaptive strategy. We thus rephrased this into "You'll have to study lots and lots if you don't want to mess up again". This option was then categorized by both judges as maladaptive (general) and as negative behavioural engagement (subtype of regulation strategy).

\subsection{Application of the Game}

The game was applied in two different schools from the South West of England to a total sample of 58 children (37 boys and 21 girls) with an age range from 8 to 11 years $(\mathrm{M}=8.81, \mathrm{SD}=1.03)$. Only children whose parents provided parental consent took part in the study. Only $10 \%$ of the children were not allowed to participate in the study. Each child was tested individually using a laptop. After creating a username and a password, they were asked to play the three levels of the games. They were reminded that they should choose the option they considered best to cheer up the character of the story. Once all children finished their participation, they were debriefed and were asked to provide some feedback about their overall experience with the game.

\section{Results}

\subsection{Scenario depicting Sadness}

In this level, most children (78\%) were capable to accurately identify sadness as the emotion felt by the character. We compared whether there were differences on reaction times (in milliseconds) between those children who accurately identified the emotion $(\mathrm{M}=10477.91, \mathrm{SD}=9142.61)$ and those who did not $(\mathrm{M}=11617.31, \mathrm{SD}=9489.54)$, finding non-significant differences between them $(\mathrm{t}(56)=.39, \mathrm{p}=$ $.70, \mathrm{~d}=0.12,95 \%$ CI $[-4675.04,6953.85])$.

We aimed at analyzing whether there was an interaction between the recognition of the emotion and the use of adaptive strategies in the three interactions. Given that all these variables were categorical, we performed hi-log-linear (hierarchical) and log-linear analyses (see Wickens, 2014). First, a saturated hierarchical log-linear (hi-log-linear) procedure was run to find the most parsimonious final model. The hi-log-linear procedure started from the saturated model which included all variables' main and 
interaction effects and thus fitted the data perfectly. In a backward-elimination procedure, interaction and main effects were removed from the saturated model, and the fit of the more parsimonious model was compared to the fit of the saturated model. The hi-log-linear procedure picked the model as the final model (1) that was more parsimonious than the saturated model and (2) whose model fit was not significantly different from the saturated model. Thus, a final model having a likelihood ratio value $\left(\chi^{2}\right)$ greater than $\mathrm{p}=.05$ is considered to be fitting. It means that the expected frequencies are close to the observed frequencies (Wickens, 2014). The final model and its fit are reported in the text. To estimate the significance of specific main or interaction effects ( $\mathrm{z}$ value parameter, partial $\chi^{2}$ ), a log-linear model was computed. For each strategy, variables included in the hi-log-linear and log-linear analyses were Emotion recognition [not correct (r), correct], Regulation interaction 1 [maladaptive (r), adaptive], Regulation interaction 2 [maladaptive (r), adaptive], and Regulation interaction 3 [maladaptive (r), adaptive] with $\mathrm{r}$ indicating the reference category of each factor for the $\mathrm{z}$ value.

The hi-log-linear analyses did not produce a significant model for any interaction of the different variables $\left(\chi^{2}=1.97, \mathrm{df}=7, \mathrm{p}=.96\right)$. Overall, most children chose adaptive strategies across the three interactions with the character. Children who chose adaptive strategies in their first interaction with the character tended to do so in their second $\left(\chi^{2}=.98, \mathrm{df}=1, \mathrm{p}=.32\right)$ and third interaction $\left(\chi^{2}=2.07, \mathrm{df}=1\right.$, $\mathrm{p}=.15$ ).

Looking at the specific strategies, in interaction 1 cognitive engagement was the most preferred (74\%), followed by affective engagement (12\%) and avoidance (12\%), and finally suppression (2\%). In Interaction 2, affective engagement was the most preferred option (55\%), followed by cognitive engagement $(31 \%)$, co-rumination $(7 \%)$ and avoidance $(7 \%)$. Finally, in interaction 3 cognitive engagement was the most preferred option (53\%) followed by affective engagement (27\%), negative affective engagement (11\%), and co-rumination (9\%).

\subsection{Scenario depicting Anger}

In this level, more than half of the children (67\%) were capable to accurately identify anger as the emotion felt by the character. We compared whether there were differences on reaction times (in milliseconds) between those children who accurately identified the emotion $(\mathrm{M}=6374.10, \mathrm{SD}=4887.86)$ and those who did not $(\mathrm{M}=6085.73, \mathrm{SD}=6326.23)$, finding non-significant differences between them $(\mathrm{t}$ $(56)=-.23, \mathrm{p}=.82, \mathrm{~d}=0.12,95 \% \mathrm{CI}[-2822.99,2246.25])$.

As in the previous level, we calculated a hi-log-linear and log-linear analyses to analyze whether there was an interaction between the recognition of the emotion and the use of adaptive strategies in the three interactions. The hi-log-linear analyses did not produce a significant model for any interaction of the different variables $\left(\chi^{2}=4.56, \mathrm{df}=6, \mathrm{p}=.60\right)$. Overall, most children chose adaptive strategies across the different interactions with the character. Children who chose adaptive strategies in their first interaction with the character tended to do so in their second $\left(\chi^{2}=.04, \mathrm{df}=1, \mathrm{p}=.85\right)$ and third interaction $\left(\chi^{2}=.32\right.$, $\mathrm{df}=1, \mathrm{p}=.57)$.

Looking at the specific strategies, in interaction 1 attention was the most preferred (66\%), followed by cognitive engagement (26\%) and negative affective engagement (5\%), and finally diminishing comparisons (3\%). In Interaction 2, attention was again the most preferred option (67\%), followed by cognitive engagement (21\%), negative affective engagement $(8 \%)$ and negative behavioural engagement (4\%). Finally, in interaction 3, cognitive engagement was the most preferred option (66\%) followed by affective engagement (23\%), negative affective engagement (10\%), and diminishing comparisons (1\%).

\subsection{Scenario depicting Fear}

In this level, more than half of the children (69\%) were capable to accurately identify fear as the emotion felt by the character. We compared whether there were differences on reaction times (in milliseconds) between those children who accurately identified the emotion $(\mathrm{M}=19156.04, \mathrm{SD}=11189.56)$ and those who did not $(M=16821.96, S D=12035.11)$, finding non-significant differences between them $(t(56)=-$ $.72, \mathrm{p}=.48, \mathrm{~d}=0.12,95 \%$ CI $[-8845.67,4177.50])$.

As in the previous levels, the hi-log-linear analyses did not produce a significant model for any interaction of the different variables $\left(\chi^{2}=9.01, \mathrm{df}=9, \mathrm{p}=.44\right)$. Overall, most children chose adaptive strategies across the different interactions with the character. Children who chose adaptive strategies in their first interaction with the character tended to do so in their second $\left(\chi^{2}=.006, \mathrm{df}=1, \mathrm{p}=.94\right)$ and third interaction $\left(\chi^{2}=1.41, \mathrm{df}=1, \mathrm{p}=.24\right)$.

Looking at the specific strategies, in interaction 1 cognitive engagement (26\%) and affective engagement (79\%) was the most preferred strategy, followed by co-rumination (12\%), and avoidance (9\%). In Interaction 2, attention was again the most preferred option (52\%), followed by co-rumination (22\%), humor (13\%), and negative affective engagement (12\%). Finally, in interaction 3 cognitive engagement 
was the most preferred option (47\%) followed by attention (33\%), diminishing comparisons (12\%), and negative affective engagement $(9 \%)$.

\subsubsection{Children's Feedback on the Game}

Children were asked to indicate in a scale from $1=$ not at all to $7=$ extremely how much they enjoyed each level of the game. Furthermore, using the same scale they also indicated how willing they were to play the game again. Finally, they were asked to write down what it could be improved/changed in the game.

Concerning children's satisfaction with the scenarios, a repeated-measures ANOVA with the different scenarios as within-subject factor showed no differences $\left(F(1,57)=.82, p=37, \eta_{p}^{2}=.014\right)$. Thus, children liked to the same extent the scenario depicting sadness $(M=5.62, S D=.79)$, anger $(M=5.93$, $\mathrm{SD}=.97)$, and fear $(\mathrm{M}=5.76, \mathrm{SD}=.99)$. Concerning, their willingness to play the game, overall children indicated they were willing to play the game again $(\mathrm{M}=5.90, \mathrm{SD}=.89)$.

Finally, concerning children's perceptions of potential improvements 28 children made some suggestions. Namely, five themes emerged: $50 \%$ of children indicated that graphics could be improved, $21 \%$ indicated that the character could speak to have a better experience, $14 \%$ indicated that the game would improve by adding music, $10 \%$ indicated that it would be great if the character could be customized, and finally one child indicated that it would be great to have more levels to play.

\section{Discussion}

Emotion regulation constitutes a key variable for children's development (e.g., Eisenberg, 2000). Therefore, having serious games that target its different domains can be extremely beneficial not only for evaluation but also for training purposes. Although some serious games have been developed, they only target the intrapersonal domain of ER overlooking the interpersonal side (e.g., Rodriguez et al., 2012). Hence, Emodiscovery, is to the best of our knowledge, the first serious game designed to address such gap.

When designing the game different aspects were taken into account. First of all, its accessibility: the game is freely available, it does not require installation and can be played on browsers or on all Windows platforms. The game is also being released on mobile devices and new releases will maintain a crossplatform compatibility and support. Regarding information security, all data are encrypted and kept on multiple cloud copies by a BaaS service. The user interface was designed to be as simple and intuitive as possible, according to the principles of usability in educational games (Amory, 2001; Shneiderman, 2010), and paying attention to possible distractions. The game is also extremely versatile and can be used for testing, training or assessing interpersonal emotional regulation, and includes tutoring aspects.

The preliminary results have shown that children may find more difficult to accurately identify the emotions of anger and fear, compared to sadness. This is in line with previous findings that showed how children (from 8 to 10 years) find it more difficult to recognize the emotions of anger and fear compared to sadness (Sauter, Panattoni, \& Happé, 2012).

Regarding the use of regulation strategies, most children used an adaptive strategy to change the emotion of the character in the story. Unlike expected, there was not an interaction between accurately identifying the emotion felt by the character and the selection of an adaptive strategy. Previous literature in interpersonal ER has suggested that in order for the agent to be able to change someone's emotions, the agent needs to understand how the other person is feeling (Zaki \& Williams, 2013). However, in our study we did not find such relationship. We believe this may be caused by the high accuracy rated to identify the character's emotions, as well as having most of the children choosing an adaptive strategy. Thus, future research should be conducted with children with emotion difficulties (e.g, children with high-functioning autism) to test whether the game may be an appropriate task to discriminate children with different levels of emotion recognition and regulation. Finally, it is important to note that children showed consistency throughout the scenarios. Thus, children who tended to choose an adaptive strategy to improve the character's sadness did choose an adaptive strategy to improve the character's anger and fear. This is in line with previous research on the use of regulation strategies that showed that overall people tend to use similar strategies across contexts (Niven, Macdonald, \& Holman, 2012).

Concerning the user experience, the overall feedback about the game was positive. The reports collected after the interaction with the platform indicate that most children enjoyed playing the game and, in particular, the majority expressed that they would play the game again. The extent and the quality of the additional suggestions collected also indicate an active engagement and involvement during the participation.

Given that Emodiscovery has the potential to provide feedback, one of its possible direct applications can be fostering children's interpersonal ER skills in educational contexts. Following Piaget's theory (1983), the feedback provided could help children to accommodate their learning on interpersonal ER, as it will 
help them to change their potential maladaptive responses to more adaptive ones. Furthermore, the existence of different levels with distinct emotions (i.e., sadness, anger, and fear) may help children to assimilate the different regulation strategies (i.e., fitting the new regulation strategies in their previous schemas of how to cheer up others) and use them across different levels. The game was designed for children between 8 to 11 years of age, which means they are capable of abstract problem solving (concrete operational stage; Piaget, 1983); hence, it is not surprising that children could represent the different emotional situations and provided in most cases a successful response by choosing an adaptive regulation strategy. Although in this first evaluation of the game we did not assess if children were capable of applying their learning (i.e., use of adaptive regulation strategies to other new contexts or levels); according to Piaget's theory (1983), learning is likely to happen as children showed active engagement. In fact, the design of the game with an online peer should be a major driving force for learning as well. Although in this application the game was administered to typically developing children, the game may have potential for children with emotion difficulties. Namely, we believe the game could be extremely beneficial for children with high-functioning autism as it may help them to improve not only their emotion recognition skills but also their interpersonal emotion regulation abilities. In this sense, there are serious games developed to train children within the Autism Spectrum Disorder to recognize others' emotions (e.g., What to choose; Grynszpan, Martin, \& Nadel, 2008) or improve their communication skills (e.g., ECHOES; Bernardini, Porayska-Pomsta, \& Smith, 2014). However, to the best of our knowledge, none of the existing serious games target interpersonal ER. Given that previous research has shown that this population may find difficulties in that domain (López-Pérez, Ambrona, \& Gummerum, 2017), we believe that such game may be extremely beneficial for this clinical population.

Although the present research constitutes only preliminary evidence about the game, we believe Emodiscovery can be extremely beneficial to advance our knowledge about children's interpersonal emotion regulation and to promote better and more tailored interventions. 
References

Akamatsu, M., \& MacKenzie, I. S. (2002). Changes in applied force to a touchpad during pointing tasks. International Journal of Industrial Ergonomics, 29(3), 171-182.

Amory, A. (2001). Building an educational adventure game: Theory, design, and lessons. Journal of Interactive Learning Research, 12(2), 249-263.

Astor, P. J., Adam, M. T., Jerčić, P., Schaaff, K., \& Weinhardt, C. (2013). Integrating biosignals into information systems: A NeuroIS tool for improving emotion regulation. Journal of Management Information Systems, 30(3), 247-278.

Batson, C. D. (2011). Altruism in humans. Oxford University Press, USA.

Bernardini, S., Porayska-Pomsta, K., \& Smith, T. J. (2014). ECHOES: An intelligent serious game for fostering social communication in children with autism. Information Sciences, 264, 41-60.

Blair, C. (2002). School readiness: Integrating cognition and emotion in a neurobiological conceptualization of children's functioning at school entry. American Psychologist, 57, 111127.

Chapman, A. L., Dixon-Gordon, K. L., \& Walters, K. N. (2011). Experiential avoidance and emotion regulation in borderline personality disorder. Journal of Rational-Emotive \& CognitiveBehavior Therapy, 29(1), 35-52.

Dunfield, K. A. (2014). A construct divided: Prosocial behavior as helping, sharing, and comforting subtypes. Frontiers in Psychology, 5, 958.

Dunfield, K. A., O’Connell, L., Kuhlmeier, V. A., \& Kelley, E. A. (2011). Examining the diversity of prosocial behaviour: Helping, sharing, and comforting in infancy. Infancy, 16, 227- 247.

Eisenberg, N. (2000). Emotion, regulation and moral development. Annual Review of Psychology, 51, 665-697.

Eisenberg, N., \& Fabes, R. (2006). Emotion regulation and children's socioemotional competence. In L. Balter \& C. Tamis-LeMonda (Eds.), Child psychology: A handbook of contemporary issues (2nd ed., pp. 357-381). New York, NY: Psychology Press.

Fagundo, A. B., Santamaría, J. J., Forcano, L., Giner- Bartolomé, C., Jiménez- Murcia, S., Sánchez, I., ... \& Lam, T. (2013). Video game therapy for emotional regulation and impulsivity control in a series of treated cases with bulimia nervosa. European Eating Disorders Review, 21(6), 493499.

Gross, J.J. (2007). Handbook of emotion regulation. New York: Guilford Press.

Gross, J.J. (2015). Emotion regulation: current status and future prospects. Psychological Inquiry, 1, 1-26.

Grynszpan, O., Martin, J. C., \& Nadel, J. (2008). Multimedia interfaces for users with high functioning autism: An empirical investigation. International Journal of Human-Computer Studies, 66(8), 628639.

Hromek, R., \& Roffey, S. (2009). Promoting social and emotional learning with Games: "It's Fun and We Learn Things". Simulation \& Gaming, 40, 626-644.

Jercic, P., Astor, P. J., Adam, M. T. P., Hilborn, O., Schaaff, K., Lindley, C., ... \& Eriksson, J. (2012). A Serious Game using Physiological Interfaces for Emotion regulation Training in the Context of Financial Decision-Making. In ECIS (Vol. 207).

Little, L. M., Kluemper, D., Nelson, D. L., \& Gooty, J. (2012). Development and validation of the Interpersonal Emotion Management Scale. Journal of Occupational and Organizational Psychology, 85, 407-420.

López-Pérez, B., Ambrona, T., \& Gummerum, M. (2017). Interpersonal emotion regulation in Asperger's syndrome and borderline personality disorder. British Journal of Clinical Psychology, 56(1), 103113.

López-Pérez, B., Wilson, E., Dellaria, G, \& Gummerum, M. (2016). Developmental Differences in Children's Interpersonal Emotion Regulation. Motivation and Emotion, 40, 767-780.

Mazefsky, C.A., Borue, Z., Day, T.N., \& Minshew, N.J. (2014). Emotion regulation patterns in adolescents with high functioning autism spectrum disorder: comparison to typically developing adolescents and association with psychiatric symptoms. Autism Research, 7, 344-354.

Natapov, D., Castellucci, S. J., \& MacKenzie, I. S. (2009, May). ISO 9241-9 evaluation of video game controllers. In Proceedings of Graphics Interface 2009 (pp. 223-230). Canadian Information Processing Society.

Niven, K., Macdonald, I., \& Holman, D. (2012). You Spin Me Right Round: Cross-Relationship Variability in Interpersonal Emotion Regulation. Frontiers in Psychology, 3, 394. http://doi.org/10.3389/fpsyg.2012.00394

Niven, K., Totterdell, P., \& Holman, D. (2009). A classification of controlled interpersonal affect regulation strategies. Emotion, 9, 498-509. 
Noor, H. A. M., Shahbodin, F., \& Pee, N. C. (2012). Serious game for autism children: Review of literature. World Academy of Science, Engineering and Technology. International Journal of Social, Behavioral, Educational, Economic, Business and Industrial Engineering, 6(4), 554559.

Parkinson, B., \& Simons, G. (2012). Worry spreads: Interpersonal transfer of problem related anxiety. Cognition and Emotion, 26, 462-479.

Parkinson, B., Simons, G., \& Niven, K. (2016). Sharing concerns: Interpersonal worry regulation in romantic couples. Emotion, 16(4), 449-458.

Piaget, J. (1983). Piaget's theory. In P. Mussen (ed). Handbook of Child Psychology. 4th edition. Vol. 1. New York: Wiley.Pioggia, G., Igliozzi, R., Sica, M. L., Ferro, M., Muratori, F., Ahluwalia, A., \& De Rossi, D. (2008). Exploring emotional and imitational android-based interactions in autistic spectrum disorders. Journal of CyberTherapy \& Rehabilitation, 1, 49-61.

Polson, M. C., \& Richardson, J. J. (Eds.). (2013). Foundations of intelligent tutoring systems. New York: Lawrence Erlbaum.

Richards, J. M., \& Gross, J. J. (2000). Emotion regulation and memory: the cognitive costs of keeping one's cool. Journal of Personality and Social Psychology, 79(3), 410-424.

Rodriguez A, Rey B, Alcaniz M, et al. (2012). GameTeen: new tools for evaluating and training emotional regulation strategies. Studies in Health Technology \& Informatics, 181, 334-338.

Samson, A. C., Wells, W. M., Phillips, J. M., Hardan, A. Y., \& Gross, J. J. (2015). Emotion regulation in autism spectrum disorder: evidence from parent interviews and children's daily diaries. Journal of Child Psychology and Psychiatry, 56(8), 903-913.

Sauter, D. A., Panattoni, C., \& Happé, F. (2013). Children's recognition of emotions from vocal cues. British Journal of Developmental Psychology, 31(1), 97-113.

Shneiderman, B. (2010). Designing the user interface: strategies for effective human-computer interaction. India: Pearson Education.

Southam-Gerow, M. A., \& Kendall, P. C. (2002). Emotion regulation and understanding: Implications for child psychopathology and therapy. Clinical Psychology Review, 22, 189-222.

Widen, S. C., \& Russell, J. A. (2003). A closer look at preschoolers' freely produced labels for facial expressions. Developmental Psychology, 39, 114-128.

Wickens, T. D. (2014). Multiway contingency tables analysis for the social sciences. Psychology Press.

Williams, M. (2007). Building genuine trust through interpersonal emotion management: A threat management model of trust and collaboration across boundaries. Academy of Management Review, $32,595-621$

Zaki, J., \& Williams, W. C. (2013). Interpersonal emotion regulation. Emotion, 13, 803-810. 
Appendix A

Example of a Full Interaction with Correct Answers (Scenario 1, Male)

Introduction:

John has a pet rabbit which he calls "Whiskers". Whiskers is brown with a white belly and enjoys eating carrots and playing with John when they return from school. One afternoon after school John opened Whiskers' cage but the rabbit did not want to play. After several days John asked his dad to take Whiskers to the vet. After being checked, the vet said to John that Whiskers was very sick.

John: Whiskers is very sick...

Initial question

Question: How is John feeling?

Player's Answer: Sadness

Owl: Well done! John is feeling sad

First interaction

John: Whiskers is very sick...

Question: Please select what you would say to John:

Player's Answer: Don't worry, Whiskers will get better

Owl: Well done! You're doing a good job at making John feel better.

Second interaction

John: I'm very sad because I can't play with Whiskers anymore...

Question: Please select what you would say to John:

Player's Answer: Don't worry you looked after Whiskers very well

Owl: Well done! You're doing a good job at making John feel better.

Third interaction

John: But I went to the vet and I don't see Whiskers getting better

Question: Please select what you would say to John:

Player's Answer: I can go to the vet with you next time

Feedback: Well done! You're doing a good job at making John feel better.

Conclusion

John: Whiskers is doing better now

Owl: Well done! You have helped John feel better about the situation 
Appendix B

Stories Piloted for the Selection of the Final Scenarios

Scenario 1: Mary/John has a pet rabbit which s/he calls "Whiskers". Whiskers is brown with a white belly and enjoys eating carrots and playing with Mary/John when they return from school. One afternoon after school Mary/John opened Whiskers' cage but the rabbit did not want to play. After several days Mary/John asked her/his dad to take Whiskers to the vet. After being checked, the vet said to Mary/John that Whiskers was very sick.

Scenario 2: Emily/Mark decided to make a card for her/him mum for mother's day. S/he wanted to make a very special card with a painting on the front with glitter, stickers and stamps. Emily/Mark has been working on the card for two weeks and s/he is very excited about giving the card to her/his mum. The day before mother's day, Emily/Mark was finishing the card at school. One of the other children in the classroom did not make mother's day card and decides to destroy Emily's/Mark's card.

Scenario 3: Sophie/Steven is going to take part in a Christmas play. S/he is one of the main characters and has spent weeks learning the lines. The teacher decided that they should perform a practice performance to the rest of the school. On the day of the practice performance Emily/Steven froze on the stage and forgot what they had to say. Children watching the play started laughing and pointing at Sophie/Steven. Next week Sophie/Steven will have to do the real performance to the whole school along with parents.

Scenario 4: Anna/Jack has been invited to a birthday party on Saturday. It's going to be a very exciting party and only ten children form the class have been invited. Anna's/Jack's mum took them shopping to get the best present and they are really excited about giving the present and going to the party. On Saturday Anna/Jack are getting ready for the party and when they are about to leave the house they receive a phone call saying the party has been cancelled.

Scenario 5: Ella/ Jonathan had been revising for a Maths test all week and s/he is really looking forward to completing the test so s/he can have time to play with her/his new computer game. Once they completed the Maths test they were very pleased with how it went and s/he thinks they are going to get a very good mark. After school the only thing that Ella/Jonathan wanted to do was to play the new computer game. Half way through the game Ella's/Jonathan's dad switched off the computer and orders her/him to tidy her/his room. The game has not been properly shut down and when Ella/Jonathan tries to play again the game does not work anymore.

Scenario 6: Charlotte/ David went shopping to get some new trainers with her/his parents and her/his younger brother on Saturday. When they got to town it was very busy as it was Carnival day so there were lots of stores and market's stands open. Whilst in town Charlotte/David thought they saw one of their classmates and tried to run after them to talk to them. After running through the crowds, Charlotte/David realized it was not her/his friend. When they turned around s/he could not see her/his family anymore. Charlotte/ David started to run around to see if s/he could spot her/his family. Fifteen minutes went by and Charlotte/ David realized that s/he could not find her/his family. 
Appendix C

Strategies Considered for the different Interaction Responses

Adaptive Interpersonal Regulation Strategies

Maladaptive Interpersonal Regulation Strategies

- Affective engagement: active commitment to the target's feelings through active listening, talking to the target, or understanding that the target has stable positive characteristics (López-Pérez, Wilson, Dellaria, \& Gummerum, 2016; Niven, Totterdell, \& Holman, 2009).

- Cognitive engagement: it involves changing the way the target thinks about a situation (Gross, 2007; López-Pérez et al., 2016; Niven et al., 2009).

- Distraction: diverting the target's attention away from an aversive event (López-Pérez et al., 2016; Niven et al., 2009)

- Humour: it implies entertaining and amusing the target to make them laugh (López-Pérez et al., 2016; Niven et al., 2009).
- Suppression: inhibition of the expressive emotional response in other (Samson, Wells, Phillips, Hardan, \& Gross, 2014)

- Co-rumination: repetitively focusing on the experience of others' negative emotion, its causes and consequences (Parkinson \& Simons, 2012; Parkinson, Simons, \& Niven, 2016).

- Avoidance: evading others' negative feelings and thoughts (Chapman, Dixon-Gordon, \& Walters, 2011).

- Diminishing comparisons: exaggerating one's own achievements and positive features to play down others' achievements (Niven et al., 2009)

- Negative behavioural engagement: highlighting others' faults and mistakes (Niven et al., 2009). 
Figure 1. User Registration Screen

Figure 2. Flow Diagram for each of the Levels.

Figure 3. Example of level interface with a female character.

Figure 4. Example of Male Character's Emotion Facial Expressions Compared to Real Human Models.

From the left: neutral facial expression, anger, fear, and sadness.

Figure 5. The Architecture and Structure of the Game, with its connection with the Game Engine and the External Database. 
Table 1

\begin{tabular}{llcc}
\hline \multirow{2}{*}{ Vser } & \multicolumn{1}{c}{ Variable recorded } & $\begin{array}{c}\text { Public } \\
\text { version }\end{array}$ & $\begin{array}{c}\text { Experimental } \\
\text { version }\end{array}$ \\
\cline { 2 - 4 } & Demographic information & Yes & Yes \\
\cline { 2 - 4 } Scenario & Number of times started & Yes & Yes \\
\cline { 2 - 4 } & $\begin{array}{l}\text { Number of scenarios played } \\
\text { Number of times completed }\end{array}$ & Yes & Yes \\
Responses given when completed the first time & Yes & Yes \\
\hline $\begin{array}{l}\text { Responses given when completed after the first } \\
\text { time }\end{array}$ & Yes & No \\
Response times (ms) & No & Yes \\
Score & & Yes & Yes \\
\hline
\end{tabular}

Variables Measured for Each User. 
Table 2

$\begin{array}{llllll}\text { Scenario } 1 & \text { Scenario } 2 & \text { Scenario } 3 & \text { Scenario } 4 & \text { Scenario } 5 & \text { Scenario } 6\end{array}$

\begin{tabular}{lcccccc} 
Happiness & $0(0 \%)$ & $0(0 \%)$ & $0(0 \%)$ & $0(0 \%)$ & $0(0 \%)$ & $0(0 \%)$ \\
Sadness & $50(83.3 \%)$ & $14(23.3 \%)$ & $9(15 \%)$ & $28(47 \%)$ & $18(33 \%)$ & $13(22 \%)$ \\
Anger & $0(0 \%)$ & $46(76.7 \%)$ & $0(0 \%)$ & $29(33 \%)$ & $32(53 \%)$ & $0(0 \%)$ \\
Surprise & $0(0 \%)$ & $0(0 \%)$ & $3(5 \%)$ & $12(20 \%)$ & $0(0 \%)$ & $12(20 \%)$ \\
Fear & $10(16.7 \%)$ & $0(0 \%)$ & $45(75 \%)$ & $0(0 \%)$ & $0(0 \%)$ & $35(58 \%)$ \\
Disgust & $0(0 \%)$ & $0(0 \%)$ & $3(5 \%)$ & $0(0 \%)$ & $10(17 \%)$ & $0(0 \%)$ \\
\hline
\end{tabular}

Frequency of Choices in the different Scenarios 


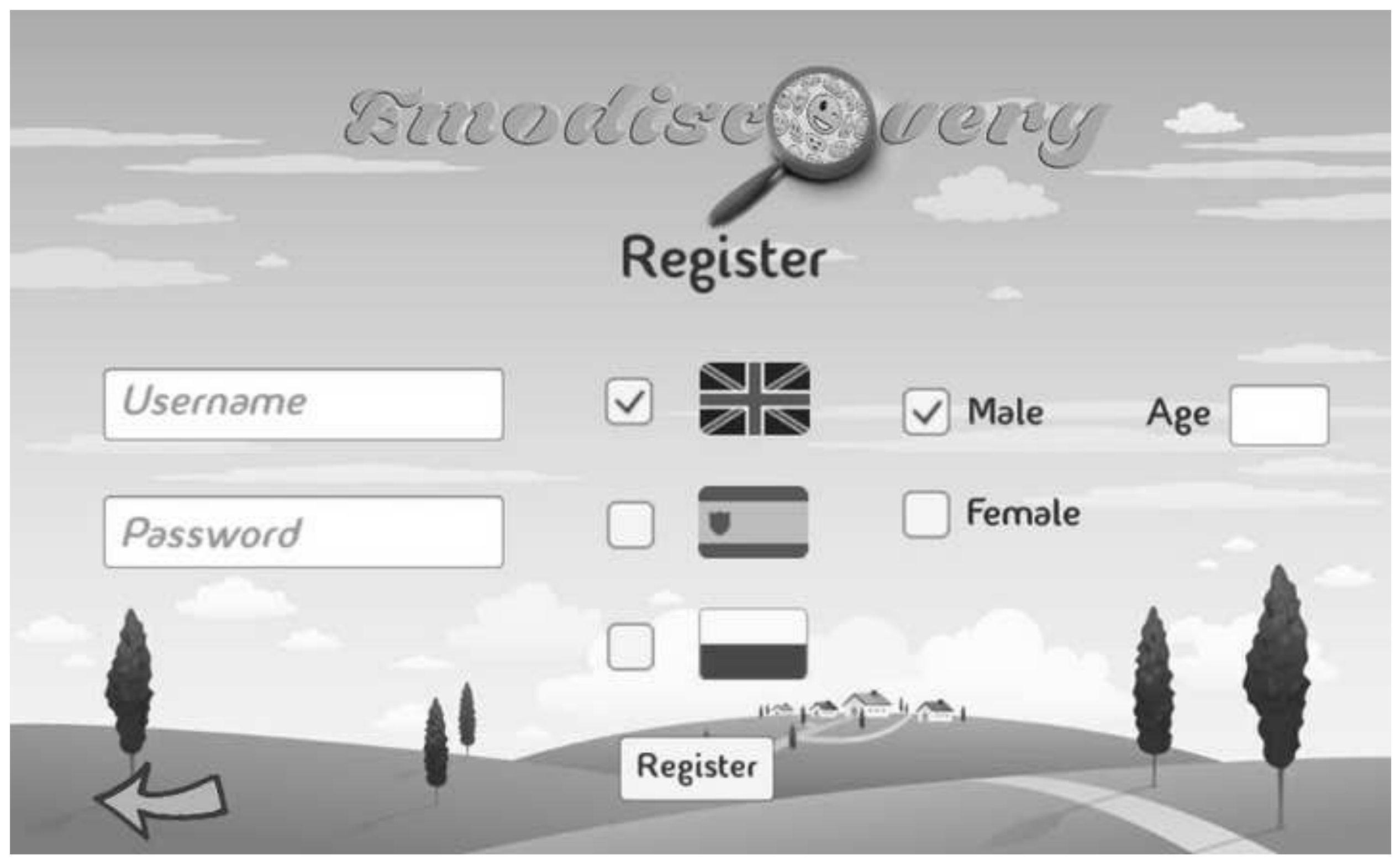

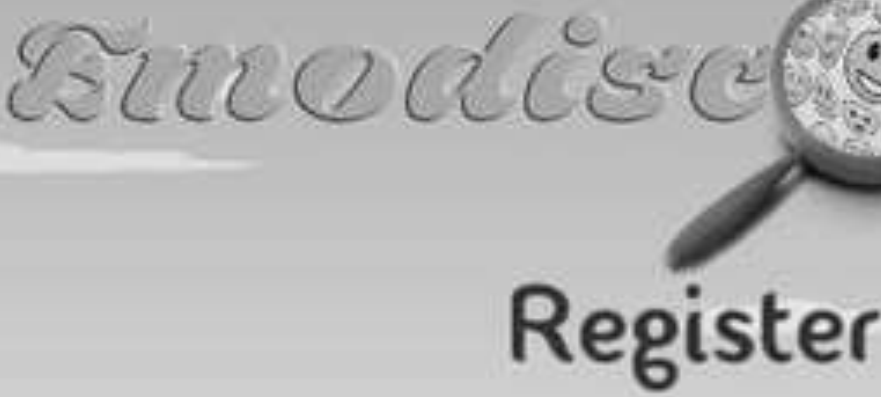

$\checkmark$ Male

Age

\section{Password}

\section{Register}

click here to downt

\section{Username}




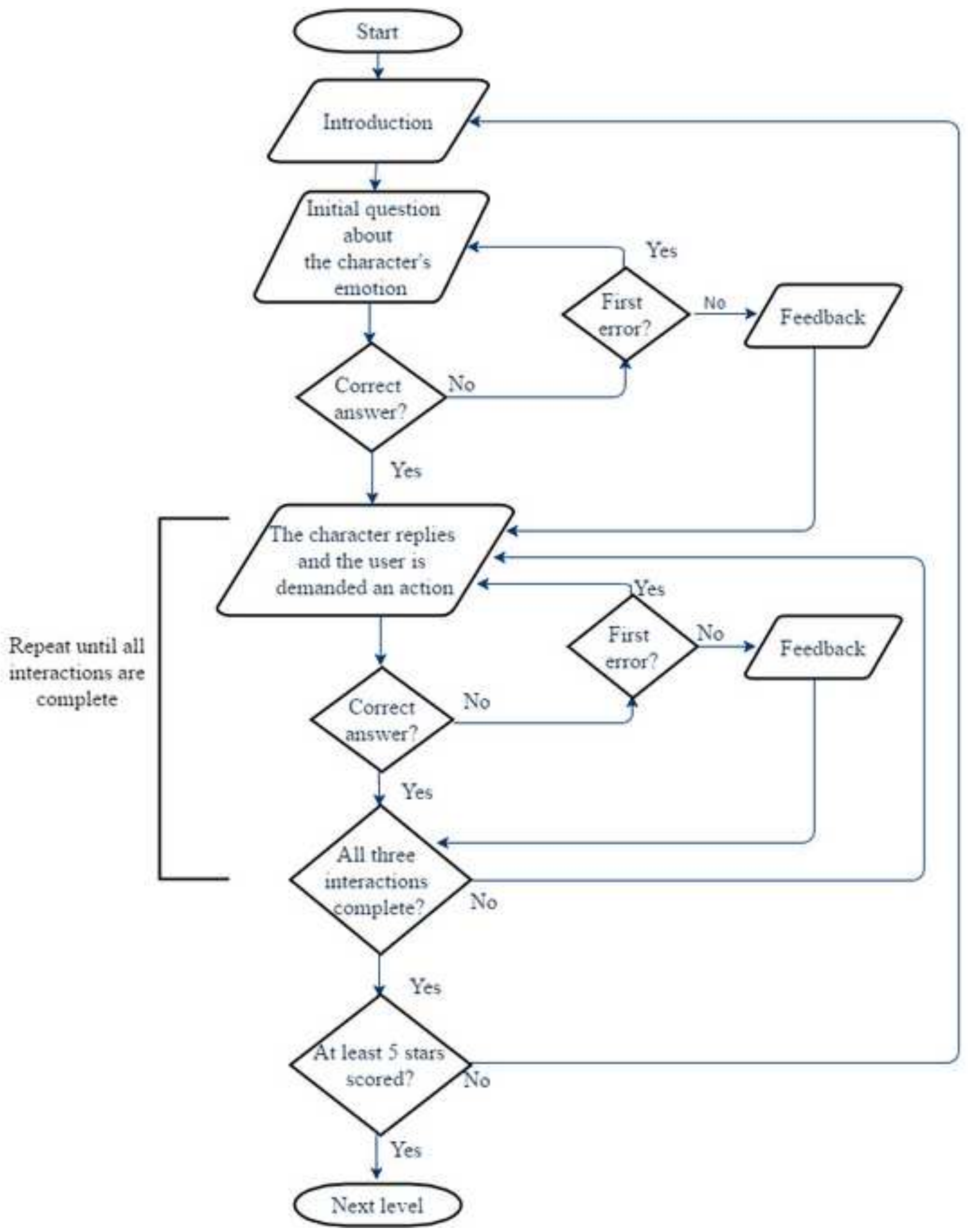




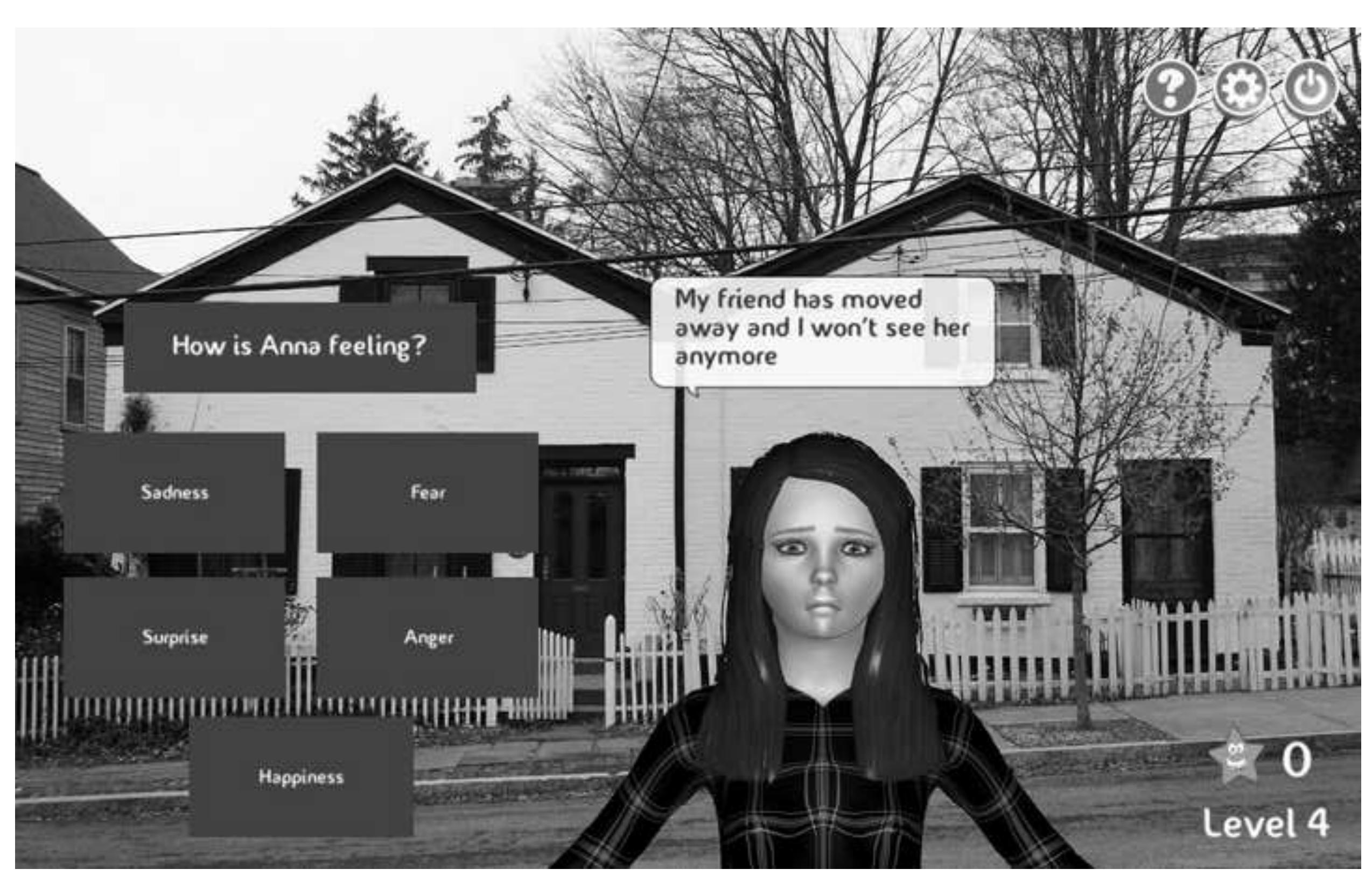



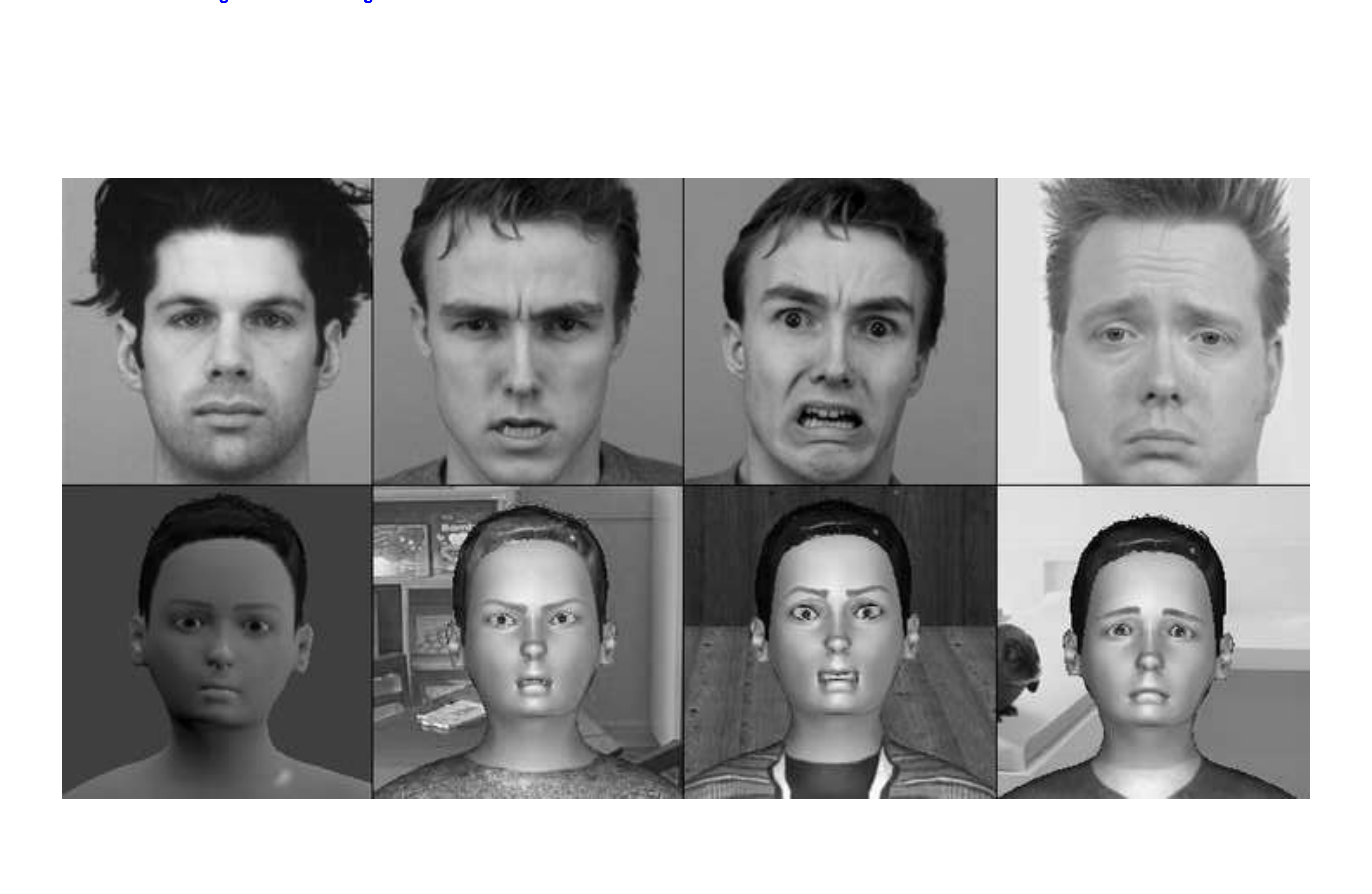
Platform

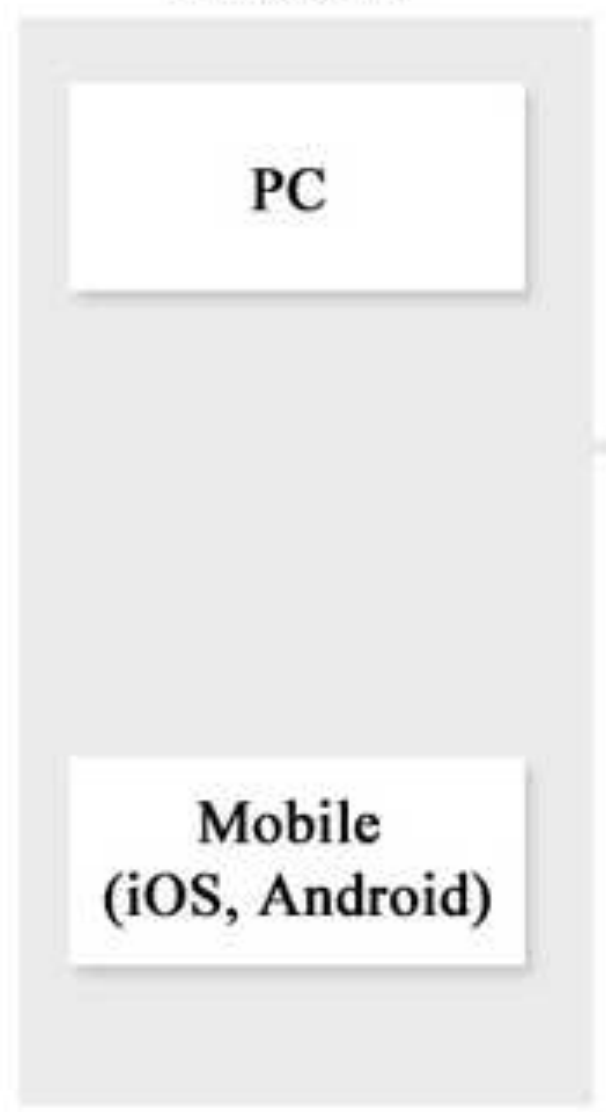

Game Loop

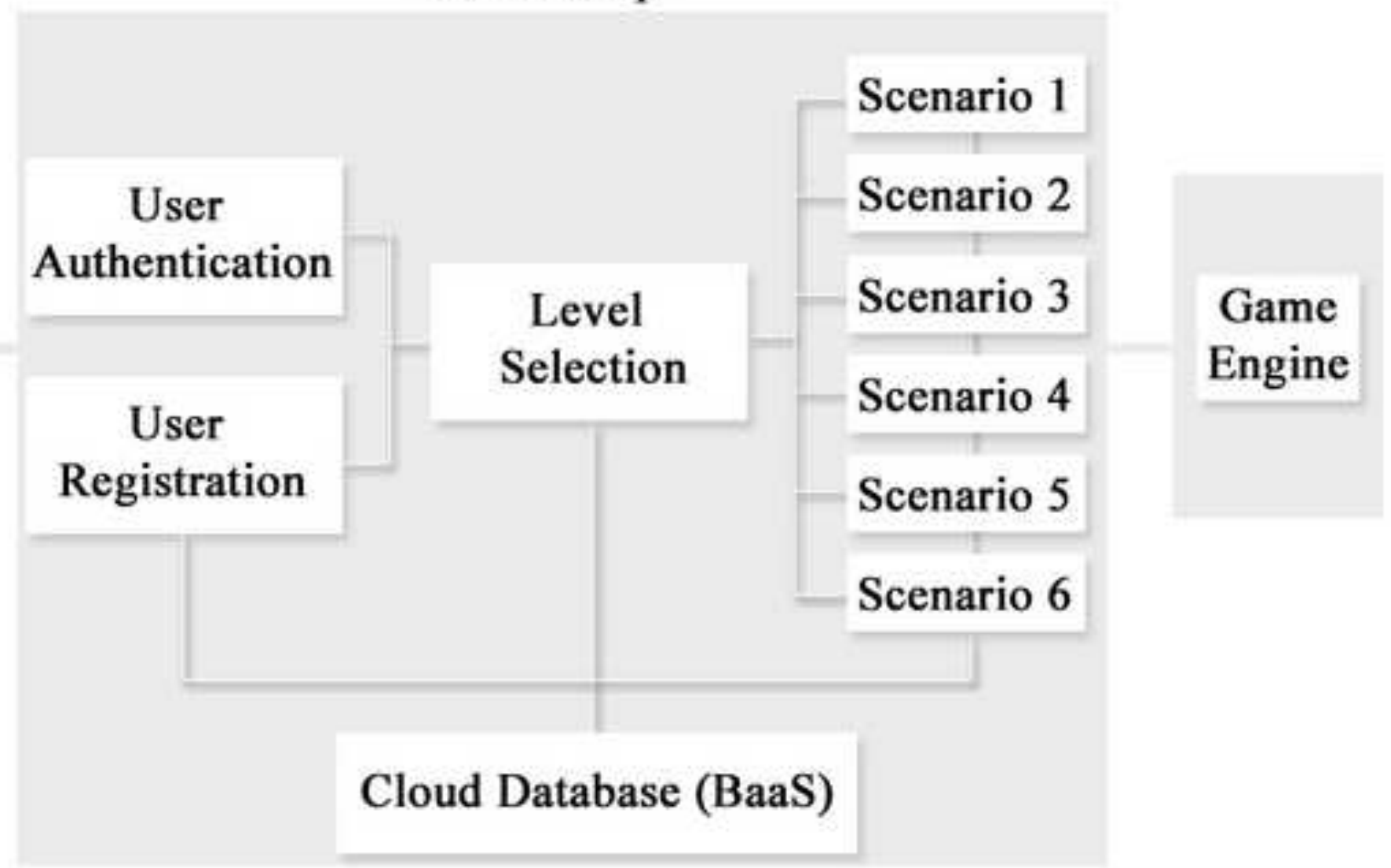


Acknowledgements

Funding: this study was funded by a Santander Postgraduate Internalization Grant (2014/2015)

awarded to the second author of the manuscript. 
Highlights

- Previous serious games have overlooked the social dimension of emotion regulation

- Emodiscovery evaluates if children (age 8-11) use maladaptive regulation strategies

- Children mainly used adaptive strategies to change the virtual character's emotion

- Children chose similar strategies across different scenarios and different emotions

- The obtained findings are discussed in terms of its possible applications 